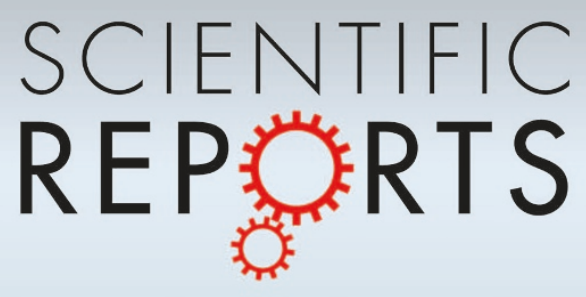

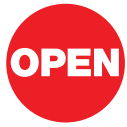

SUBJECT AREAS: STRUCTURAL PROPERTIES

THERMOELECTRICS

STRUCTURE OF SOLIDS AND

LIQUIDS

MECHANICAL ENGINEERING

Received

9 October 2012

Accepted

6 December 2012

Published

31 January 2013

Correspondence and requests for materials should be addressed to

N.Y. (imyangnuo@ tongii.edu.cn) or B.L. (phylibw@nus.edu.sg)

\section{Reduction of Thermal Conductivity by Nanoscale 3D Phononic Crystal}

\author{
Lina Yang ${ }^{1}$, Nuo Yang ${ }^{2} \&$ Baowen Li1 ${ }^{1,2,3}$
}

'Department of Physics and Centre for Computational Science and Engineering, National University of Singapore, Singapore 1 17542, Republic of Singapore, ${ }^{2}$ Center for Phononics and Thermal Energy Science, School of Physical Science and Engineering, Tongii University, 200092 Shanghai, People's Republic of China, ${ }^{3}$ NUS Graduate School for Integrative Sciences and Engineering, National University of Singapore, Singapore 117456, Republic of Singapore.

We studied how the period length and the mass ratio affect the thermal conductivity of isotopic nanoscale three-dimensional (3D) phononic crystal of Si. Simulation results by equilibrium molecular dynamics show isotopic nanoscale 3D phononic crystals can significantly reduce the thermal conductivity of bulk $\mathrm{Si}$ at high temperature $(1000 \mathrm{~K})$, which leads to a larger ZT than unity. The thermal conductivity decreases as the period length and mass ratio increases. The phonon dispersion curves show an obvious decrease of group velocities in 3D phononic crystals. The phonon's localization and band gap is also clearly observed in spectra of normalized inverse participation ratio in nanoscale 3D phononic crystal.

hermoelectric materials are important for generating electricity from waste heat and being used as solid-state Peltier coolers. The performance of thermoelectric materials depends on the figure of merit $(\mathrm{ZT})^{1}, \mathrm{ZT}=$ $\mathrm{S}^{2} \sigma \mathrm{T} / \kappa$, where $\mathrm{S}, \mathrm{T}, \sigma$, and $\kappa$ are the Seebeck coefficient, absolute temperature, electrical conductivity and total thermal conductivity, respectively. ZT can be increased by increasing $\mathrm{S}$ or $\sigma$, or decreasing $\kappa$. However, it is difficult to improve ZT in conventional materials. First, simple increase S for general materials will lead to a simultaneous decrease in $\sigma^{1,2}$. Also, an increase in $\sigma$ leads to a comparable increase in the electronic contribution to $\kappa^{1,2}$.

An alternative way to increase ZT is to reduce the thermal conductivity without affecting electronic property ${ }^{1,3}$. Moreover, ultra-low thermal conductivity is also required to prevent the back-flow of heat from hot end to cool end. Therefore, reduction of thermal conductivity is crucial in thermoelectric application.

Phononic crystals are constructed by a periodic array of scattering inclusions distributed in a host material. Due to its periodic change of the density and/or elastic constants, phononic crystals exhibit phononic band gaps ${ }^{4}$. This remarkable property is very different from those of the conventional materials and can be engineered to achieve new functionalities. A special one-dimensional phononic crystal is the superlattice, one dimensional periodic arrangement of two different materials. It is demonstrated that superlattice crystals are effective to achieve very low thermal conductivity ${ }^{5-11}$. Superlattices have been extensively studied to design thermoelectric materials with high ZT. Preliminary works show that there is a minimum value of thermal conductivity in the direction perpendicular to the planes of superlattice when the period length is reduced to nanoscale ${ }^{12-14}$.

Recently, it is demonstrated experimentally that the Si nanomesh film, a two-dimensional (2D) phononic crystal, exhibited low thermal conductivity ${ }^{15}$ by modification of phonon band structure. Single crystalline Si by phononic crystal patterning in $2 \mathrm{D}$ has a smaller value of thermal conductivity $(\sim 6 \mathrm{~W} / \mathrm{m}-\mathrm{K})$ than bulk Si because of the low group velocities and the coherent phononic effects ${ }^{16}$. It is predicted that atomic-scale $3 \mathrm{D}$ phononic crystal of Ge quantum-dot in Si has very low thermal conductivity in all three spatial directions ${ }^{17}$. The thermal conductivity is reduced by several orders of magnitude compared with bulk Si. This reduction of thermal conductivity is due to the reduction in group velocities and multiple scattering of particle-like phonons.

In this letter, we study the thermal conductivity of nanoscale 3D silicon phononic crystal. The 3D crystal consists of ${ }^{28} \mathrm{Si}$ atoms and "isotopes" ${ }^{\mathrm{M}} \mathrm{Si}$ atoms which have the same properties as ${ }^{28} \mathrm{Si}$ except the mass, where $\mathrm{M}$ is the atomic mass of the isotope of $\mathrm{Si}$. The mass ratio, $\mathrm{R}$, is defined as $\mathrm{R}=\mathrm{M} / 28$. The $3 \mathrm{D}$ isotopic phononic crystal could also be named as 3D superlattice because different material arranged periodically in three spatial directions.

We find the 3D isotopic phononic crystal has the ability to flatten phonon dispersion curves compared with that of bulk Si and it could show band gaps when properly arranged. We studied how the period length and the mass ratio affect the thermal conductivity of the 3D phononic crystal. The phonon dispersion curves and inverse participation ratio are also computed to understand the mechanism of the reduction of thermal conductivity. 
On the other hand, the scatterings of isotopic doping could significantly reduce the lattice thermal conductivity without much reduction of the electrical conductivity ${ }^{1,14}$. As a result, the thermal conductivity of $3 \mathrm{D}$ isotopic phononic crystal has a quite low value, which can lead to a larger ZT than unity.

\section{Results}

Fig. 1 shows the structures of the isotopic nanoscale 3D phononic crystal, where ${ }^{28} \mathrm{Si}$ and ${ }^{\mathrm{M}} \mathrm{Si}$ atoms are assembled periodically in three spatial directions. Fig. 1(a)-(d) shows the structures of 3D phononic crystals with different period lengths, corresponding to $1.09 \mathrm{~nm}$, $2.17 \mathrm{~nm}, 3.26 \mathrm{~nm}$ and $6.52 \mathrm{~nm}$, respectively. The volume of simulation cell is $12 \times 12 \times 12$ unit $^{3}$ ( 1 unit is $0.543 \mathrm{~nm}$ ), which has 13,824 atoms.

There is finite size effect in calculating thermal conductivity when the simulation cell is not big enough ${ }^{18,19}$. As shown in Fig. 2, we calculated thermal conductivity of isotopic nanoscale 3D phononic crystal with different size of simulation cell by equilibrium molecular dynamic (EMD) method at $1000 \mathrm{~K}$. The period length is set as 2 units and the mass ratio is set as 2 . The calculated value of thermal conductivity converges when the side length of cubic simulation cell is larger than 10 units. To overcome the finite size effect on the calculated thermal conductivity, we use the side length of simulation cell as 12 units in the following simulations.

For comparison with the thermal conductivity of pure ${ }^{28} \mathrm{Si}$ at $1000 \mathrm{~K}$, we also calculate its value as $50 \pm 2 \mathrm{~W} / \mathrm{m}-\mathrm{K}$ (the dash dot line in Fig. 3(a)). Our result is comparable to Schelling et al.'s results of $\mathrm{MD}$ simulation, $61 \mathrm{~W} / \mathrm{m}-\mathrm{K}^{18}$. However, $\mathrm{MD}$ results can not exactly coincide with the experimental value of ${ }^{28} \mathrm{Si}$ at $1000 \mathrm{~K}^{20}$, around $30 \mathrm{~W} / \mathrm{m}-\mathrm{K}$, because of the inaccuracies of semi-empirical potentials and the impurity of the sample in measurements. This non-coincidence has little effect on the comparing MD results calculated using same potential parameters.

We calculated the thermal conductivity of $3 \mathrm{D}$ phononic crystal with different period length, where the mass ratio $\mathrm{R}$ is 2 . As shown in Fig. 3(a), the thermal conductivity rapidly decreases as the period length increases. The smallest value of thermal conductivity is $2.14 \mathrm{~W} / \mathrm{m}-\mathrm{K}$, which is only $4.3 \%$ of pure ${ }^{28} \mathrm{Si}$ calculated by EMD method. Simkin and Mahan show ${ }^{12}$ that increasing period length (a)

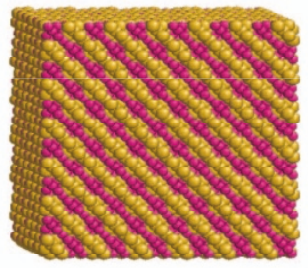

(c)

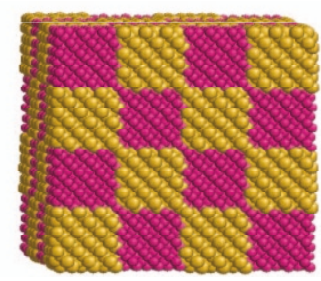

(b)

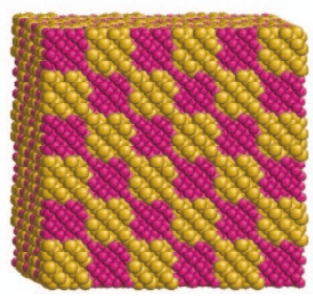

(d)

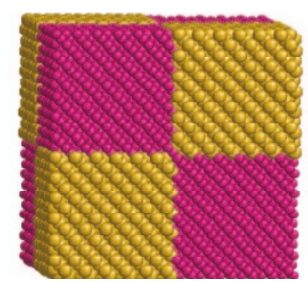

Figure $1 \mid$ The structures of the isotopic nanoscale 3D phononic crystals three dimensional periodic arrangements of ${ }^{28} \mathrm{Si}$ and ${ }^{\mathrm{M}} \mathrm{Si}$ atoms. From (a) to (d), the period lengths of those 3D phononic crystals are 2, 4, 6 and 12 units, respectively. The lattice constant is $0.543 \mathrm{~nm}$, that is, 1 unit represents $0.543 \mathrm{~nm}$. In simulations, the periodic boundary condition is applied in all three directions.

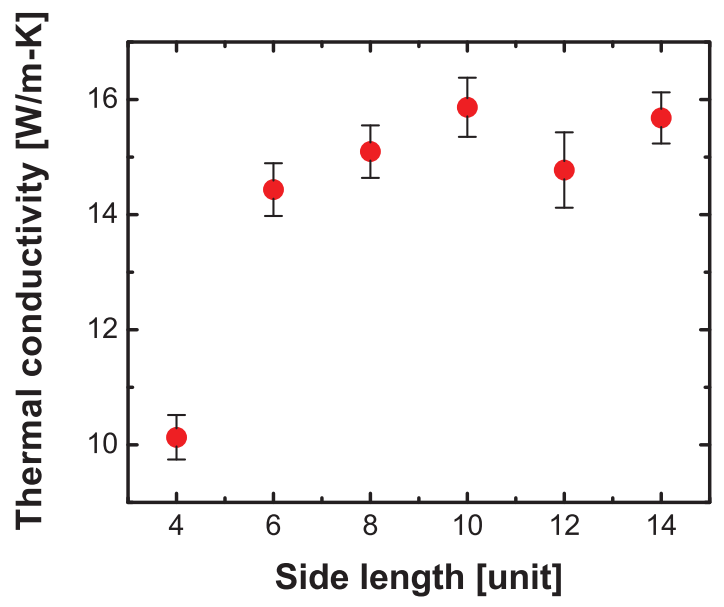

Figure $2 \mid$ Thermal conductivity versus the side length of simulation cell. The mass ratio of the $3 \mathrm{D}$ isotopic phononic crystal is 2 and the period length is 2 units. The error bars are calculated from 16 simulations with different initial conditions. All values are calculated at $1000 \mathrm{~K}$ which is larger than the Debye temperature, $\mathrm{T}_{\mathrm{D}}$, of $\mathrm{Si}(\sim 658 \mathrm{~K})$.

may increase the amount of band folding and decrease the average velocity in the superlattice, resulting in a decrease of thermal conductivity. The phonon mean free path of $\mathrm{Si}$ is around $60 \mathrm{~nm}$ at $1000 \mathrm{~K}^{18}$, which is much longer than the period length in our simulation. The tendency of thermal conductivity in Fig. 3(a) is consistent with Simkin and Manhan's results when the phonon mean free path is larger than period length ${ }^{12}$.

Another way to modulate the phonon transport in the $3 \mathrm{D}$ phononic crystal is to vary the mass. The mass of impurity atoms could perturb the phonon density of state and phonon dispersion curves, which can affect the group velocities.

Fig. 3(b) shows the dependence of thermal conductivity on the mass ratio, where the period length is 12 units. Our results indicate that thermal conductivity rapidly decreases as the mass ratio increases from 1 to 6 . The heaviest $\mathrm{Si}$ isotope atoms produced is ${ }^{43} \mathrm{Si}^{21}$. where the mass ratio $\mathrm{R}$ corresponds to 1.5 . The value of thermal conductivity is $4.2 \mathrm{~W} / \mathrm{m}-\mathrm{K}$, that is, 8.4 percent of pure ${ }^{28} \mathrm{Si}(50 \mathrm{~W} / \mathrm{m}-\mathrm{K})$.

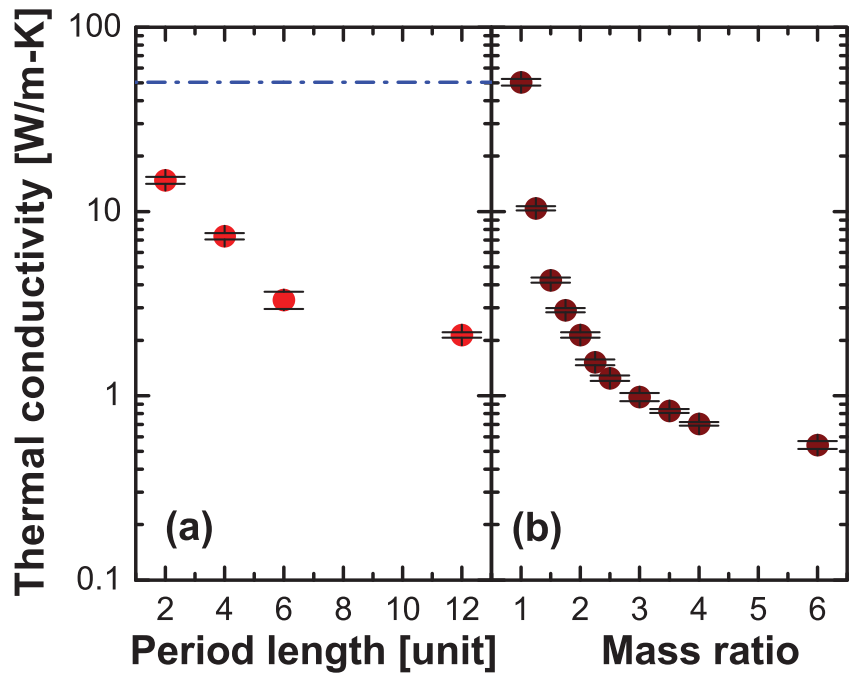

Figure 3 | (a) Thermal conductivity versus the period length of isotopic nanoscale $3 \mathrm{D}$ phononic crystal of $\mathrm{Si}$. The mass ratio is 2 . The dash dot line corresponds to the molecular dynamic result of thermal conductivity of pure ${ }^{28} \mathrm{Si}$. (b) Thermal conductivity versus the mass ratio of isotopic nanoscale 3D phononic crystals of Si. The period length is 12 units. All values are calculated at $1000 \mathrm{~K}$. 
Artificial $\mathrm{Si}$ isotopic atoms are used here to explore the mass influence on thermal transport and show the trend of large mass effects. The smallest value of thermal conductivity is $0.54 \mathrm{~W} / \mathrm{m}-\mathrm{K}$ (when $\mathrm{R}$ is 6), which is only $1.1 \%$ of pure ${ }^{28} \mathrm{Si}$. Artificial ${ }^{\mathrm{M}} \mathrm{Si}$ atoms can be looked as other atoms, such as ${ }^{54} \mathrm{Fe}^{22}$. When there are other kind atoms, the system is more complicated. That is, the mass is not the only factor involved. The bond strength and lattice relaxations must play a role, which is not studied in this letter.

In a ${ }^{28} \mathrm{Si}_{182}{ }^{\mathrm{M}} \mathrm{Si}_{10}$ quasi-1D supercell with $10{ }^{\mathrm{M}} \mathrm{Si}$ atoms (5\%) randomly distributed, Gibbons and Estreicher ${ }^{22}$ found the thermal conductivity decreased first and reached a minimum when the mass ratio was "two", and then the thermal conductivity increased as the increase of M. However, they stated that they cannot comment about the reasons for this minimum and do not know if the factor "two" remains valid for concentrations other than 5\%. Different from randomly distributed, ${ }^{\mathrm{M}} \mathrm{Si}$ in $3 \mathrm{D}$ phononic crystal is periodic distributed in ${ }^{28} \mathrm{Si}$. The concentration of ${ }^{\mathrm{M}} \mathrm{Si}(50 \%)$ is much bigger than $5 \%$. Our results show that the thermal conductivity of $3 \mathrm{D}$ phononic crystal decreases monotonously with increase of $\mathrm{M}$. This is coincidence of the monotonous decrease of group velocities (Fig. 4(b)).

As shown above, changing the mass of impurity atoms and the period lengths are two effective ways in modulating the thermal conductivity. To find the mechanism in the decrease of thermal conductivity of $3 \mathrm{D}$ phononic crystal, the phonon dispersion curves are calculated through classical lattice dynamics. We calculated the dispersion curves by general utility lattice program (GULP) $)^{23}$, and Stillinger-Weber potential ${ }^{24}$ which is the same atom interaction as in our MD simulation.

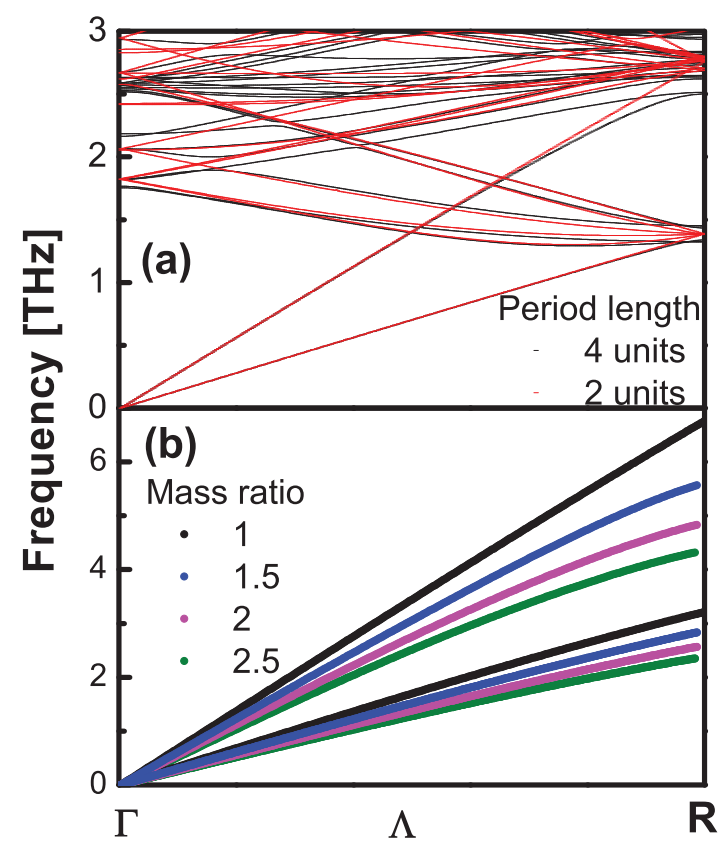

Figure $4 \mid$ (a) Acoustic and partial optical branches along the $[1,1,1]$ direction. Dispersion curves in red (black) correspond to the nanoscale 3D phononic crystal with 2 (4) unit cells in period length. The mass ratios are 2. It is clearly shown close to $\mathrm{R}$ point that the group velocities decrease as the period length increases, which causes the reduction of the thermal conductivity. (b) Acoustic branches along the $[1,1,1]$ direction. The mass ratio of $3 \mathrm{D}$ phononic crystals changes from 1 to 2.5. Different color is referred to different mass ratio. The period lengths of $3 \mathrm{D}$ phononic crystals are kept same, 2 units. The dispersion curves are affected by the mass of impurity atoms and the group velocities decrease as the mass of impurity atoms increase, which contributes to the decrease of thermal conductivity.
Fig. 4(a) shows acoustic branches and partial optical branches of the dispersion curves of the $3 \mathrm{D}$ phononic crystal, where period lengths are different, and the mass ratio is the same. The Brillouin zone with 2 units in period length is eight times as large as that with 4 units in period length. In calculating the dispersion curves of structure with 2 units in period length, we use a larger unit cell whose size is as the same as that of structure with 4 units in period length.

As the optical phonons contribute less to the thermal conductivity due to the lower group velocities, we focused on the acoustic phonons. It is clearly shown in Fig. 4(a) close to $\mathrm{R}$ point that the group velocities decrease as the period length increase, which causes the reduction of the thermal conductivity (shown in Fig. 3(a)). Fig. 4(b) shows acoustic branches of the $3 \mathrm{D}$ phononic crystal with different mass ratios, where the period length is 2 units. The dispersion curves are affected by the mass of impurity atoms and the group velocities decrease as the mass of impurity atoms increase, which contributes to the decrease of thermal conductivity (shown in Fig. 3(b)).

To understand more about the underlying physical mechanism of thermal conductivity reduction, we carry out a vibration eigen-mode analysis on 3D phononic crystals. The mode localization can be qualitatively characterized by the normalized inverse participation ratio (NIPR) ${ }^{25}$. The NIPR for phonon mode $\mathrm{k}$ is defined through the normalized eigenvector $\mathrm{u}_{\mathrm{k}}$

$$
P_{k}^{-1}=N \cdot \sum_{i=1}^{N}\left(\sum_{\alpha=1}^{3} u_{i \alpha, k}^{2}\right)^{2}
$$

(a)
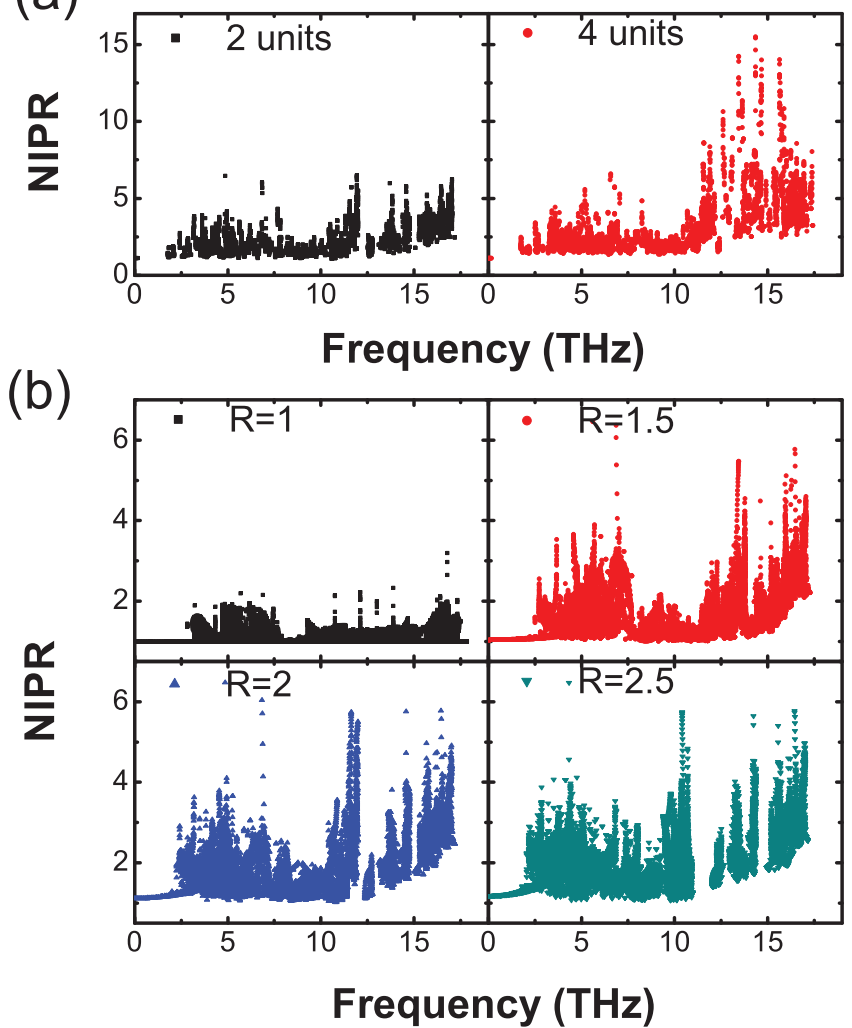

Figure $5 \mid$ The normalized inverse participation ratio (NIPR) spectra. NIPR is calculated based on Eq. (1). The larger of the value of NIPR the more localized of a phonon mode. (a) NIPR spectra of 3D phononic crystals with different period length. The left and right panels are corresponding to $3 \mathrm{D}$ phononic crystals with 2 unit and 4 unit period length, respectively. The mass ratios are the same as 2. (b) NIPR spectra of $3 \mathrm{D}$ phononic crystals with different mass ratio, $\mathrm{R}$. The period lengths are the same as 2 units. The upper left panel $(\mathrm{R}=1)$ corresponds to pure Si. 
where $N$ is the total number of atoms. When there are less atoms participating in the motion, the phonon mode has a larger NIPR value. For example, NIPR is $N$ when there is only one atom vibrates in the localized mode. When all atoms participate in the motion, NIPR is calculated out as 1 . That is, the larger of the value of NIPR the more localized of a phonon mode.

Fig. 5(a) shows the NIPR spectra of 3D phononic crystal with two different period lengths, where the mass ratio $R$ is 2 . Obviously, the NIPR for 3D phononic crystal with 4 units in period length has larger values than that with 2 units in period length. That is, there are more modes localized in $3 \mathrm{D}$ phononic crystal with 4 units in period length, which also leads to a reduction of its thermal conductivity. Fig. 5(b) shows the NIPR spectra of 3D phononic crystal with different mass ratio $\mathrm{R}$, where the period length is 2 units. The values of NIPR for $\mathrm{R}=1.5, \mathrm{R}=2$ and $\mathrm{R}$ $=2.5$ are close to each other and larger than pure $\mathrm{Si}(\mathrm{R}=1)$, which show that the isotopic atoms could cause more localizations. In Fig. 5(b), band gaps appear in the high frequency part $(>12 \mathrm{THz})$ of spectra for $\mathrm{R}=2$ and $\mathrm{R}=2.5$, and the band gaps for $\mathrm{R}=2.5$ are wider than those for $\mathrm{R}=2$. That is, the phonons with frequency in the range of band gaps cannot exist in the $3 \mathrm{D}$ phononic crystal. When $\mathrm{R}$ is smaller than 1.5 (corresponding to ${ }^{43} \mathrm{Si}$ ), band gaps are not observed in $3 \mathrm{D}$ isotopic phononic crystal. So, band gaps are minor effects when the mass difference is not big enough.

Besides decreasing group velocity and phonon modes localization, interface disorder is another factor in reducing the thermal conductivity for superlattices ${ }^{11}$ and phononic crystal ${ }^{16}$. In our simulation, ${ }^{28} \mathrm{Si}$ and ${ }^{\mathrm{M}} \mathrm{Si}$ atoms has a perfect interface which do not include the disorder effect. There will be a further deduction in thermal conductivity after considering the interface disorder. So, the disorder effect will be studied in the future work.

\section{Discussion}

Using the Green-Kubo method, we have calculated thermal conductivities of isotopic nanoscale 3D phononic crystals, where ${ }^{28} \mathrm{Si}$ and ${ }^{\mathrm{M}} \mathrm{Si}$ atoms are assembled periodically in the three directions. Results show that the thermal conductivity decreases as the increasing of period length from $1 \mathrm{~nm}$ to $6 \mathrm{~nm}$. The thermal conductivity of structure with $6 \mathrm{~nm}$ period length and 2 in mass ratio is $2.14 \mathrm{~W} / \mathrm{m}-\mathrm{K}$ at $1000 \mathrm{~K}$, which is only $4.3 \%$ of pure ${ }^{28} \mathrm{Si}$ and can lead to a larger ZT than unity.

Moreover, the thermal conductivity rapidly decreases as the mass ratio increases. The phonon localizations and bandgaps at high frequency in the $3 \mathrm{D}$ phononic crystal are shown clearly in the spectra of the normalized inverse participation ratio. The appearance of band gaps blocks a range of frequency of phonon modes and flattens phonon dispersion curves. The phonon dispersion curves show the phonon group velocities decrease in $3 \mathrm{D}$ phononic crystal. In a word, the decrease of thermal conductivity in $3 \mathrm{D}$ phononic crystal is attributed to both the decrease of group velocities and the localization.

Thermal conductivity is mainly contributed from acoustic phonons. If the bandgaps of the 3D phononic crystals can exist at low frequencies, there will be greater reduction of the thermal conductivity. However, manipulating band gaps to low frequencies in nanoscale $3 \mathrm{D}$ phononic crystal is a challenging work which is worthy of effort in the future. There are advances in obtaining the nanoscale $2 \mathrm{D}$ phononic crystal. However, the limitation in fabricating nanoscale $3 \mathrm{D}$ phononic crystal is still challenging nowadays.

\section{Methods}

The Green-Kubo method, equilibrium molecular dynamics (MD), is employed to calculate the thermal conductivities of $3 \mathrm{D}$ phononic crystal at $1000 \mathrm{~K}$. MD simulation is a popular method in calculating thermal conductivity at high temperature ${ }^{18,26,27}$. In this letter, we focus on the thermal conductivity of $3 \mathrm{D}$ phononic crystal at $1000 \mathrm{~K}$, which is larger than the Debye temperature, $\mathrm{T}_{\mathrm{D}}$, of $\mathrm{Si}(\sim 658 \mathrm{~K})^{28}$.
In simulations, the periodic boundary condition is applied in all three directions To derive the force term, we use Stillinger-Weber (SW) potential for $\mathrm{Si}^{24}$, which includes both two-body and three-body potential terms. The SW potential has been used widely to study the thermal properties of Si bulk material ${ }^{14,26,29}$ for its accurate fit for experimental results on the thermal expansion coefficients. The heat current is defined as ${ }^{18}$

$$
\vec{J}_{l}(t)=\sum_{i} \vec{v}_{i} \varepsilon_{i}+\frac{1}{2} \sum_{i j} \vec{r}_{i j}\left(\vec{F}_{i j} \cdot \vec{v}_{i}\right)+\sum_{i j k} \vec{r}_{i j}\left(\vec{F}_{j}(i j k) \cdot \vec{v}_{j}\right)
$$

where $\vec{F}_{i j}$ and $\vec{F}_{i j k}$ denote the two-body and three-body force, respectively. Thermal conductivity is calculated from the Green-Kubo formula ${ }^{30}$

$$
\kappa=\frac{1}{3 k_{B} T^{2} V} \int_{0}^{\infty}<\vec{J}(\tau) \cdot \vec{J}(0)>d \tau
$$

where $\kappa$ is thermal conductivity, $k_{B}$ is the Boltzmann constant, $V$ is the system volume, $T$ is the temperature, and the angular bracket denotes an ensemble average.

Generally, the temperature in $\mathrm{MD}$ simulation, $T_{M D}$, is calculated from the kinetic energy of atoms according to the Boltzmann distribution:

$$
\langle E\rangle=\sum_{i=1}^{N} \frac{1}{2} m v_{i}^{2}=\frac{3}{2} N k_{B} T_{M D}
$$

where $\langle E\rangle$ is the total kinetic energy, $v_{i}$ is the velocity, $m$ is the atomic mass, $N$ is the number of particles in the system, and $k_{B}$ is the Boltzmann constant. This equation is valid at high temperature ( $T \gg T_{D}, T_{D}$ is the Debye temperature).

Numerically, velocity Verlet algorithm is employed to integrate equations of motion, and each MD step is set as 1.0 fs. Firstly, canonical ensemble MD with langevin heat reservoir runs for $2^{20}$ steps to equilibrate the whole system at $1000 \mathrm{~K}$ Then, microcanonical ensemble (NVE) MD runs for another $2^{25}$ steps (33.5 ns). Meanwhile, heat current is recorded at each step. At the end, the thermal conductivity is calculated by Eq. (3). In the calculation of thermal conductivity, the integration is from zero to a cut-off time which is determined by "first avalanche" method ${ }^{31}$. The final result is averaged over sixteen realizations with different initial conditions to satisfy ergodicity.

1. Chen, G., Dresselhaus, M. S., Dresselhaus, G., Fleurial, J. P. \& Caillat, T. Recent developments in thermoelectric materials. Int. Mater. Rev. 48, 45-66 (2003).

2. Hicks, L. D. \& Dresselhaus, M. S. Thermoelectric figure of merit of a onedimensional conductor. Phys. Rev. B 47, 16631-16634 (1993).

3. Majumdar, A. Thermoelectricity in Semiconductor Nanostructures. Science 303, 777-778 (2004).

4. Gorishnyy, T., Maldovan, M., Ullal, C. \& Thomas, E. Sound ideas. Phys. World 18, 24-29 (2005)

5. Yang, R. \& Chen, G. Thermal conductivity modeling of periodic two-dimensional nanocomposites. Phys. Rev. B 69, 195316 (2004).

6. Kim, W. et al. Thermal Conductivity Reduction and Thermoelectric Figure of Merit Increase by Embedding Nanoparticles in Crystalline Semiconductors. Phys. Rev. Lett. 96, 045901 (2006).

7. Hochbaum, A. I. et al. Enhanced thermoelectric performance of rough silicon nanowires. Nature 451, 163 (2008).

8. Boukai, A. I. et al. Silicon nanowires as efficient thermoelectric materials. Nature 451, 168-171 (2008).

9. Yang, N., Ni, X., Jiang, J.-W. \& Li, B. How does folding modulate thermal conductivity of graphene? Appl. Phys. Lett. 100, 093107-093104 (2012).

10. Hyldgaard, P. \& Mahan, G. D. Phonon superlattice transport. Phys. Rev. B 56, 10754 (1997)

11. Chen, G. \& Neagu, M. Thermal conductivity and heat transfer in superlattices. Appl. Phys. Lett. 71, 2761-2763 (1997).

12. Simkin, M. V. \& Mahan, G. D. Minimum Thermal Conductivity of Superlattices. Phys. Rev. Lett. 84, 927 (2000).

13. Venkatasubramanian, R. Lattice thermal conductivity reduction and phonon localizationlike behavior in superlattice structures. Phys. Rev. B 61, 3091-3097 (2000).

14. Yang, N., Zhang, G. \& Li, B. Ultralow therma conductivity of isotope-doped silicon nanowires. Nano Lett. 8, 276-280 (2008)

15. Yu, J. K., Mitrovic, S., Tham, D., Varghese, J. \& Heath, J. R. Reduction of thermal conductivity in phononic nanomesh structures. Nat. Nanotechnol. 5, 718-721 (2010).

16. Hopkins, P. E. et al. Reduction in the Thermal Conductivity of Single Crystalline Silicon by Phononic Crystal Patterning. Nano Lett. 11, 107-112 (2011).

17. Gillet, J. N., Chalopin, Y. \& Volz, S. Atomic-Scale Three-Dimensional Phononic Crystals With a Very Low Thermal Conductivity to Design Crystalline Thermoelectric Devices. J. Heat Transf.-Trans. ASME 131, 043206 (2009).

18. Schelling, P. K., Phillpot, S. R. \& Keblinski, P. Comparison of atomic-level simulation methods for computing thermal conductivity. Phys. Rev. B 65, 144306 (2002). 
19. Che, J., Cagin, T., Deng, W. \& Goddard Iii, W. A. Thermal conductivity of diamond and related materials from molecular dynamics simulations. J. Chem Phys. 113, 6888-6900 (2000).

20. Glassbrenner, C. J. \& Slack, G. A. Thermal Conductivity of Silicon and Germanium from $3{ }^{\circ} \mathrm{K}$ to the Melting Point. Phys. Rev. 134, A1058-A1069 (1964)

21. Notani, M. et al. New neutron-rich isotopes, $34 \mathrm{Ne}, 37 \mathrm{Na}$ and $43 \mathrm{Si}$, produced by fragmentation of a $64 \mathrm{~A} \mathrm{MeV} \mathrm{48Ca} \mathrm{beam.} \mathrm{Phys.} \mathrm{Lett.} \mathrm{B} \mathrm{542,} \mathrm{49-54} \mathrm{(2002).}$

22. Gibbons, T. M. \& Estreicher, S. K. Impact of Impurities on the Thermal Conductivity of Semiconductor Nanostructures: First-Principles Theory. Phys. Rev. Lett. 102, 255502 (2009)

23. Gale, J. D. GULP: A computer program for the symmetry-adapted simulation of solids. J. Chem. Soc., Faraday Trans. 93, 629-637 (1997).

24. Stillinger, F. H. \& Weber, T. A. Computer simulation of local order in condensed phases of silicon. Phys. Rev. B 31, 5262 (1985).

25. Jiang, J.-W. \& Wang, J.-S. Conditions for the existence of phonon localized edgemodes. Phys. Rev. B 81, 174117 (2010).

26. Yang, N., Zhang, G. \& Li, B. Violation of Fourier's law and anomalous heat diffusion in silicon nanowires. Nano Today 5, 85-90 (2010).

27. Esfarjani, K., Chen, G. \& Stokes, H. T. Heat transport in silicon from firstprinciples calculations. Phys. Rev. B 84, 085204 (2011).

28. Holland, M. G. Analysis of Lattice Thermal Conductivity. Phys. Rev. 132, 2461-2471 (1963).

29. Volz, S. G. \& Chen, G. Molecular dynamics simulation of thermal conductivity of silicon nanowires. Appl. Phys. Lett. 75, 2056-2058 (1999).

30. Kubo, R., Toda, M. \& Hashitsume, N. Vol. 31 Springer Series in Solid-State Sciences 146-202 (Springer Berlin Heidelberg, 1991).
31. Chen, J., Zhang, G. \& Li, B. How to improve the accuracy of equilibrium molecular dynamics for computation of thermal conductivity? Phys. Lett. A 374, 2392-2396 (2010).

\section{Acknowledgements}

This work was supported in part by the grant from the Asian Office of Aerospace R\&D of the US Air Force (AOARD-114018) (LY and BL), the startup fund from Tongji University (NY and BL) and the National Natural Science Foundation of China (Grant No. 11204216) (NY). NY and LY are grateful to Jin-Wu Jiang (Bauhaus- University Weimar), Jie Chen (NUS), Sha Liu (NUS), and Lifa Zhang (NUS) for useful discussions.

\section{Author contributions}

L.Y. and N.Y. carried out the numerical simulations and data analysis. B.L. and N.Y. supervised the projects. All authors discussed the results and contributed to writing the manuscript.

\section{Additional information}

Competing financial interests: The authors declare no competing financial interests.

License: This work is licensed under a Creative Commons

Attribution-NonCommercial-No Derivs 3.0 Unported License. To view a copy of this license, visit http://creativecommons.org/licenses/by-nc-nd/3.0/

How to cite this article: Yang, L., Yang, N. \& Li, B. Reduction of Thermal Conductivity by Nanoscale 3D Phononic Crystal. Sci. Rep. 3, 1143; DOI:10.1038/srep01143 (2013). 